\title{
Hábitos de Búsqueda y Escaneo de Información sobre Salud en Adultos Chilenos
}

\author{
Macarena Sofía Peña y Lillo Arayasa \\ Universidad Diego Portales (Chile)
}

Palabras clave

Exposición incidental, diferencias socioeconómicas, información sobre salud, auto-cuidado de salud, audiencias

\begin{abstract}
Resumen
Este estudio explora las conductas de búsqueda y escaneo de información sobre la práctica regular de actividad física y el consumo de frutas y vegetales en una muestra no probabilística de 125 hombres y 125 mujeres adultos a través de encuestas cara a cara en la ciudad de Santiago, Chile. La búsqueda corresponde a esfuerzos activos del individuo por obtener información, mientras que el escaneo concierne a la exposición incidental a información sobre salud. Las fuentes de información examinadas fueron médicos u otros profesionales de la salud, familiares o amigos, internet, televisión o radio y diarios o revistas. Se analizaron frecuencias, tablas cruzadas y regresiones logísticas. Se encontró que en la muestra el escaneo era más frecuente que la búsqueda de información para algunas de las fuentes estudiadas y que la búsqueda no era más prevalente en ningún caso. Las mujeres tienden a escanear y buscar más que los hombres y se advierten diferencias por nivel socioeconómico en la búsqueda de información sobre salud a través de internet y diarios o revistas.
\end{abstract}




\section{Habits of Seeking and Scanning Health Information in Chilean Adults}

\author{
Key words \\ Incidental exposure, \\ socioeconomic \\ differences, health \\ information, \\ health self-care, \\ audiences.
}

\begin{abstract}
This study explores the behaviors of seeking and scanning information about the regular practice of physical activity and fruit and vegetable consumption in a no probabilistic sample of 125 adult males and 125 adult females through a face to face survey in the city of Santiago, Chile. Seeking corresponds to active efforts done by the individual to obtain information; while scanning concerns incidental exposure to health information. The examined sources of information were doctors or other health professionals, relatives or friends, internet, television or radio and newspapers or magazines. Frequencies, crosstabs and logistic regressions were analyzed. It was found that in the sample scanning was more prevalent than seeking for some of the studied sources and that seeking was not more prevalent in any case. Women tend to scan and seek more than men and differences for socioeconomic level in health information seeking through the internet and newspapers and magazines were found.
\end{abstract}

\section{Cómo citar el artículo}

Peña y Lillo Arayasa, M. S. (2016). Hábitos de búsqueda y escaneo de información sobre salud en adultos chilenos. Revista de Comunicación y Salud, Vol. 6, pp. 29-42.

DOI: http://doi.org/10.35669/revistadecomunicacionysalud.2016.6.29-42 
La información sobre salud que recibimos de nuestro entorno, tanto a través de los medios de comunicación como de nuestras redes interpersonales, tiene el potencial de guiar nuestra conducta, ayudarnos a tomar decisiones e incluso empujarnos a realizar cambios de comportamiento (Lee, Zhao, \& Pena-y-Lillo, 2016) y adoptar hábitos de vida saludable (Ramírez et al., 2013). Por esta razón, es importante conocer cómo las personas que no enfrentan un problema de salud en particular, es decir, las personas sanas, obtienen o se relacionan con la información de salud que está disponible en su entorno.

Este estudio explora dos modos de obtener información sobre salud: la búsqueda activa y el escaneo o exposición incidental. Mientras la primera se caracteriza por ser un acto deliberado en el que la persona hace un esfuerzo activo por obtener información, en la segunda la persona recibe información de su entorno como parte de su consumo rutinario de información y sin hacer esfuerzos concretos por obtenerla. El foco de este estudio es la búsqueda y escaneo de información sobre la práctica regular de actividad física y el consumo de frutas y vegetales en una muestra no probabilística de adultos de la ciudad de Santiago, en Chile. El propósito de este estudio es conocer la prevalencia de búsqueda en comparación con la del escaneo de información sobre ambas conductas de salud, así como determinar si existen diferencias en estas prácticas relacionadas con el sexo y/o el nivel socioeconómico de la persona que pudieran contribuir a la brecha de acceso a información sobre estos hábitos de auto-cuidado.

\section{Marco teórico}

Los medios de comunicación, ya sea a través de productos informativos o de entretención, están permanentemente ofreciendo información relativa a salud y hábitos de auto-cuidado (Lee, 2009). Además, las conversaciones interpersonales, las interacciones a través de redes sociales, y las búsquedas en internet pueden contener información sobre enfermedades, síntomas, hábitos de salud, entre otros. Estas instancias también contribuyen a la exposición de los individuos a información de salud y a las decisiones que tomen (Niederdeppe et al., 2007), por lo que es fundamental ampliar las fronteras del estudio de los mecanismos a través de los cuales las personas acceden a información sobre salud para incluir la exposición que ocurre a través de los medios de comunicación, internet y fuentes interpersonales.

Las conductas de búsqueda y escaneo de información son dos maneras distintas de relacionarse con ésta. La búsqueda de información ha sido definida como la realización de "esfuerzos activos para obtener información fuera de los patrones normales de exposición a fuentes mediadas o interpersonales" (Niederdeppe et al., 2007, p. 155, traducción propia), mientras que escaneo corresponde a "la adquisición de información que ocurre dentro de los patrones rutinarios de exposición a fuentes mediadas e interpersonales que puede ser recordada por el individuo fácilmente" (Niederdeppe et al., 2007, p. 154, traducción propia).

Cuando se estudian las conductas de búsqueda y escaneo de información, diversas fuentes pueden ser consideradas, por ejemplo, profesionales de la salud, medios masivos de comunicación, internet y otros. Los investigadores que propusieron estos conceptos argumentan que todas las fuentes pueden ser usadas para búsqueda y escaneo, indistintamente, a pesar de las diferentes características de cada fuente (Niederdeppe et al., 2007). Estudios empíricos, sin embargo, han revelado que ciertas fuentes son preferidas por las personas para realizar búsqueda de información, mientras que otras son más probablemente escaneadas. Específicamente, los medios masivos son más escaneados que internet o las fuentes interpersonales. Por ejemplo, cuando una persona está viendo las noticias por televisión y aparece una nota sobre estrategias para perder peso no necesariamente realiza una búsqueda intencionada 
de esa información, pero de todas maneras se expone a ella. Las fuentes interpersonales, tales como profesionales de la salud, amigos y familiares, por su parte, son por lo general más utilizadas para la búsqueda activa (Askelson, Campo, \& Carter, 2011), así como también los libros e internet.

La exposición a mensajes de salud está asociada a la práctica de hábitos saludables, pues a través de la búsqueda y el escaneo las personas aprenden estrategias para practicar conductas específicas, por ejemplo, conocen los beneficios de una conducta, lo que puede ser una importante motivación para ponerla en práctica. Además, la búsqueda y escaneo de un individuo en su red interpersonal puede redundar en una percepción de apoyo social necesario para incentivar la realización de la conducta, y pueden ser recordatorios de las normas sociales que son prevalentes en un grupo respecto de la conducta de salud (Hornik et al., 2013; Ramírez et al., 2013).

Estudios empíricos han encontrado asociaciones de naturaleza transversal entre conductas de búsqueda y escaneo de información y la realización de comportamientos preventivos en salud, tales como el consumo de frutas y verduras y el ejercicio físico (Kelly et al., 2010; Ramanadhan \& Viswanath, 2006). También se ha encontrado vínculos entre búsqueda y escaneo y la realización de una colonoscopía para detectar cáncer de colon y recto (Kelly et al., 2010; Shim, Kelly \& Hornik., 2006). Estudios longitudinales han mostrado que la búsqueda de información predice el cuidado de la dieta, el ejercicio físico y el consumo de frutas y verduras (Ramírez et al., 2013), mientras que el escaneo predice la actividad física y la mantención de un consumo de frutas y verduras saludables, lo que implica que la exposición por escaneo podría servir para reforzar antecedentes que un individuo ya maneja sobre la importancia de las conductas saludables (Hornik et al., 2013). Además, la búsqueda y el escaneo de información de distintas fuentes podrían tener diferentes efectos en las conductas de salud de las personas. Por ejemplo, Beaudoin y Hong (2011) descubrieron que las personas que buscaban información en los periódicos tenían hábitos más saludables de consumo de frutas y vegetales, mientras que aquellos que buscaban información en la televisión solían consumir más bebidas azucaradas.

\subsection{Diferencias socioeconómicas y de género en exposición a información de salud}

La posición social de una persona tiene una gran injerencia en las chances que dicha persona tenga de estar expuesta a mensajes de salud o de hábitos saludables. Cuando pensamos en la cobertura de los medios de comunicación sobre temas de salud o contenido en línea sobre estos asuntos, es esperable que el acceso de las personas a estos canales esté influenciado por sus recursos monetarios. Los estudios tempranos de la brecha digital confirmaron que existían grandes diferencias entre personas de altos y bajos ingresos en el acceso a tecnologías de la información e internet. En gran medida estas diferencias se explican por el costo de comprar un computador y pagar por una suscripción a internet. De manera similar, el acceso a información especializada a través de la suscripción a revistas y televisión por cable está determinado por la capacidad de pagar por ellas.

El acceso a atención médica también depende en gran medida del nivel de ingreso de una persona o su familia. En Chile, aun cuando el número de beneficiarios del sistema público de salud sobrepasa considerablemente el número de aquellos en el sistema privado, de los 29.996 médicos registrados en el país solo el $43 \%$ está contratado en servicios públicos (Guillou, Carabantes, \& Bustos, 2011). En consecuencia, el número de pacientes por cada médico en el sector privado se estima en 276, mientras que en el sistema público es de 920 (Guillou et al., 2011). En ese contexto, los pacientes de menores ingresos tienen menores posibilidades de ver un 
médico y de establecer con el proveedor de salud una relación en la cual éste entregue consejos y los aliente a practicar hábitos de auto-cuidado.

Los estudios en búsqueda y escaneo de información han encontrado diferencias en estas conductas entre los distintos grupos sociales, en particular dados por el nivel educacional. En general, los estudios tienden a centrarse en el nivel educacional como marcador de NSE, a pesar de que este concepto engloba otros indicadores, tales como el ingreso y la ocupación, debido a la reticencia de los individuos a entregar estos antecedentes (ver Lee, Ramírez, Lewis, Gray, \& Hornik, 2012). Los estudios que han examinado la influencia del nivel educacional han mostrado que los segmentos más educados de la población buscan información de fuentes más diversas comparados con miembros de grupos menos educados (Ramanadhan \& Viswanath, 2006). Además, las personas más educadas tienen a buscar y escanear más información (Shim et al., 2006) a través de diferentes medios (Viswanath \& Ackerson, 2011), mientras que las personas de grupos sociales más desaventajados tienen menores oportunidades para buscar información sobre salud en internet (Shim, 2008). También se han encontrado diferencias por nivel educacional en las fuentes preferidas para obtener información de salud (Beaudoin \& Hong, 2011) y los temas sobre los cuales se busca información (Galarce et al., 2011).

Además de las diferencias por nivel educacional, estudios previos en Estados Unidos han demostrado que las mujeres tienden a buscar información sobre salud más frecuentemente que los hombres (Kelly et al., 2010, Rutten, Squiers, \& Hesse, 2006) y las conductas de escaneo son también más comunes entre las mujeres (Kelly et al., 2010).

La gran mayoría de los estudios sobre búsqueda y escaneo de información sobre salud se ha realizado en Estados Unidos, en especial utilizando la base de datos de la Health Information National Trends Survey (HINTS), una encuesta nacionalmente representativa que se concentra en la adquisición de información sobre el cáncer y conductas asociadas a su prevención (por ejemplo: Rutten, Squiers, \& Hesse, 2006). Poco se sabe acerca de la adquisición de información sobre hábitos de vida saludable en países en vías de desarrollo y en poblaciones sin problemas de salud específicos.

\section{Hipótesis y preguntas de investigación}

De acuerdo a la literatura revisada en la sección previa, se proponen las siguientes hipótesis y preguntas de investigación:

Pregunta 1: ¿En qué fuentes es más prevalente la búsqueda y en cuáles el escaneo de información sobre hábitos saludables?

H1: Las costumbres de búsqueda y escaneo serán más prevalentes en las mujeres que en los hombres.

H2: A mayor nivel socioeconómico, más prevalentes serán las conductas de búsqueda y escaneo.

Pregunta 2: ¿Existen diferencias entre hombres y mujeres en búsqueda y escaneo dentro de un segmento socioeconómico específico?

Pregunta 3: ¿Es el escaneo más prevalente que la búsqueda en todos los segmentos socioeconómicos y/o entre hombres y mujeres?

\section{Método}

\subsection{Participantes y procedimiento}

Los datos para este estudio fueron obtenidos a través de una encuesta intercept de tipo transversal encargada a la empresa de estudios de opinión pública Demoscópica. La muestra comprende 250 personas mayores de 18 años, sin ningún 
diagnóstico de salud en particular, en Santiago de Chile, estratificadas en los niveles socioeconómicos (NSE) medio (C2), medio-bajo (C3) y bajo (D). Un 50\% de la muestra corresponde a mujeres. El trabajo de campo se realizó el jueves 15 de octubre de 2015 en nueve puntos de la ciudad de Santiago, donde los entrevistados fueron abordados para responder la encuesta cara a cara.

\subsection{Mediciones}

Al comienzo de la entrevista se aplicó un filtro para determinar el NSE del encuestado. A cada entrevistado se le consultó sobre la profesión o trabajo y el nivel educacional de la persona que aporta el principal ingreso del hogar. La ocupación fue consultada como una pregunta abierta, pero luego los encuestadores debieron asignarla a una de seis categorías: (1) trabajos menores ocasionales; (2) obrero no calificado, oficio menor, servicio doméstico con contrato; (3) obrero calificado, capataz, junior, micro-empresario; (4) empleado administrativo medio y bajo, vendedor, jefe de sección; (5) ejecutivo medio, profesional, mediano empresario; (6) alto ejecutivo, director grandes empresas, profesión liberal independiente, gran empresario. El nivel educacional consistía en siete categorías (1) básica incompleta o menos; (2) básica completa; (3) media incompleta o media técnica completa; (4) media completa o técnica superior incompleta; (5) universitaria incompleta o técnica superior completa; (6) universitaria completa y (7) postgrado. Utilizando ambas respuestas, el entrevistador debía clasificar a los encuestados en uno de los tres NSE en que se enfoca este estudio.

El encuestador clasificó a cada entrevistado como hombre o mujer y le consultó por su edad a la fecha de la encuesta. Con el fin de medir los comportamientos de salud de los entrevistados se les consultó en cuántos de los últimos 7 días habían practicado alguna actividad física que les haya provocado un aumento de los latidos del corazón por al menos 20 minutos (respuestas de 0 a 7). Además, se les consultó por su promedio de consumo diario de frutas y de verduras en la última semana. Las opciones de respuesta eran (1) no comí; (2) una vez al día; (3) 2 veces al día; (4) 3 veces al día; (5) 4 veces al día; (6) 5 o más veces al día. Se computó la suma del consumo de frutas y de verduras para crear una sola variable indicadora del consumo de frutas y vegetales diario. Estas mediciones de actividad física y consumo de frutas y vegetales han sido utilizadas en estudios previos sobre la búsqueda y escaneo de información, como el estudio de Hornik et al. (2013).

Para medir búsqueda y escaneo se utilizó el formato de preguntas propuesto y validado por Kelly, Niederdeppe y Hornik (2009). A los encuestados se les leyó el siguiente texto a modo introductorio: "algunas veces las personas buscan activamente información sobre temas de salud, por ejemplo, haciendo preguntas o una búsqueda activa, mientras que otras veces se topan, sin querer, con esta información". Luego se les realizó la pregunta: "en los últimos 3 meses, ¿ha buscado información sobre la práctica de actividad física de alguna de las siguientes fuentes?". Los entrevistados recibieron una tarjeta que indicaba cada una de las fuentes examinadas: (1) médico u otro profesional de la salud; (2) familiar, amigo o compañero de trabajo; (3) internet; (4) televisión o radio; y (5) diario o revista. Las opciones de respuesta fueron sí y no. La misma pregunta y mecanismo fue utilizado en el caso del consumo de frutas $y$ verduras.

Inmediatamente después de las preguntas sobre búsqueda, los encuestadores hicieron la siguiente pregunta para medir escaneo: "en los últimos 3 meses, ¿se ha topado con información sobre la práctica de actividad física de alguna de las siguientes fuentes?". En este caso los participantes tenían a mano la misma tarjeta con las cinco fuentes descrita arriba. El mismo procedimiento fue utilizado para el escaneo de información sobre consumo de frutas y verduras. 


\subsection{Análisis}

Para responder la pregunta de investigación 1 se examinaron las tablas de contingencia con la prevalencia de las conductas de búsqueda y escaneo para cada una de las fuentes y cada uno de los temas examinados. Se computó la estadística de McNemar, que sirve para probar diferencias entre las frecuencias marginales de una tabla. Luego, las frecuencias de búsqueda y escaneo fueron examinadas para derivar las conclusiones.

Para probar las hipótesis 1 y 2 se realizaron regresiones logísticas con búsqueda y escaneo de información para cada uno de los temas y cada una de las fuentes examinadas como variables dependientes; sexo y NSE como variables independientes categóricas, y edad y práctica de actividad física o consumo de frutas y verduras como variables de control. Para responder la pregunta 2 se creó una nueva variable con 6 categorías que correspondía a la interacción entre NSE y sexo. Luego se examinaron las tablas de contingencia y se obtuvo la estadística Chi cuadrado para cada una de dichas tablas. En cuanto a la pregunta 3, se examinaron las tablas de contingencia para cada segmento socioeconómico y para hombres y mujeres, comparando la tasa de búsqueda y escaneo para cada una de las fuentes.

\section{Resultados}

La edad promedio de los encuestados fue de 37,9 años $(D E=13,8)$ con un mínimo de 18 y un máximo de 78 años. El promedio de días de la semana en que los entrevistados declararon practicar alguna actividad física fue de 1,72 días ( $D E=2.09$ ). Un $9,6 \%$ de los entrevistados declaró no haber comido frutas la semana anterior; un $40,0 \%$ declaró haber comido frutas una vez al día; un $28,0 \%$, dos veces al día; y un $22,4 \%$, tres o más veces al día. Respecto al consumo de verduras, un 4,8\% declaró no haber consumido; un 49,6\% declaró haber comido una vez al día; un 25,6\% declaró haber consumido dos veces al día; y un $20,0 \%$, tres o más veces al día.

En relación a la información sobre la práctica de actividad física, un 16,8\% de la muestra declaró haber buscado activamente información a través de consultas a un médico u otro profesional de la salud en los últimos tres meses; un $24,8 \%$ buscó mediante consultas a familiares, amigos o compañeros de trabajo; un 43,6\% utilizó internet; un $19,2 \%$ la televisión o radio y un $7,6 \%$, diarios o revistas. Sobre el mismo tema, un 15,6\% declaró haber tenido escaneo o exposición incidental a través de un médico u otro profesional de la salud; un $32,4 \%$ de un familiar, amigo o compañero de trabajo; un 42,4\% en internet; un $33,2 \%$ en televisión o radio y un $16,4 \%$ en diarios 0 revistas.

Para información acerca del consumo de frutas y verduras, un 16,4\% declaró buscar información a través de un médico u otro profesional de la salud; un $24,0 \%$ a través de familiares, amigos o compañeros de trabajo, un 41,6\% a través de internet, un $24,4 \%$ de la televisión o radio y un $9,2 \%$ de diarios o revistas. En cuanto al escaneo sobre el consumo de frutas y verduras, un 17,6\% señaló haber recibido información de parte de un médico u otro profesional de la salud; un $27,6 \%$ de familiares, amigos o compañeros de trabajo, un $43,6 \%$ de internet; un $40,4 \%$ de la televisión o radio y un $18,8 \%$ de diarios o revistas. A continuación se presentan los resultados para cada una de las hipótesis y preguntas de investigación.

Las figuras 1 y 2 muestran las frecuencias de búsqueda y escaneo para actividad física y consumo de frutas y verduras respectivamente. Cuando se examina la obtención de información sobre práctica de actividad física (AF) y consumo de frutas y verduras (FV) desde la televisión o radio (AF: $p<, 001 ; \mathrm{FV}: p<, 001)$ y desde diarios 
o revistas (AF: $p<, 001 ; \mathrm{FV}: p<, 001$ ) se observa que el escaneo o exposición incidental es más recurrente que la búsqueda intencionada. Con respecto a la información obtenida de parte de familiares, amigos o compañeros de trabajo, el escaneo es más prevalente que la búsqueda solo en el caso de la información sobre actividad física $(p<, 05)$ pero no sobre consumo de frutas y verduras. Para la obtención de información a través de internet y a través de un médico u otro profesional de la salud no hay diferencias estadísticamente significativas entre escaneo y búsqueda para ninguno de los dos temas examinados. En ninguno de los casos examinados la búsqueda de información resulta significativamente más prevalente que la exposición incidental.

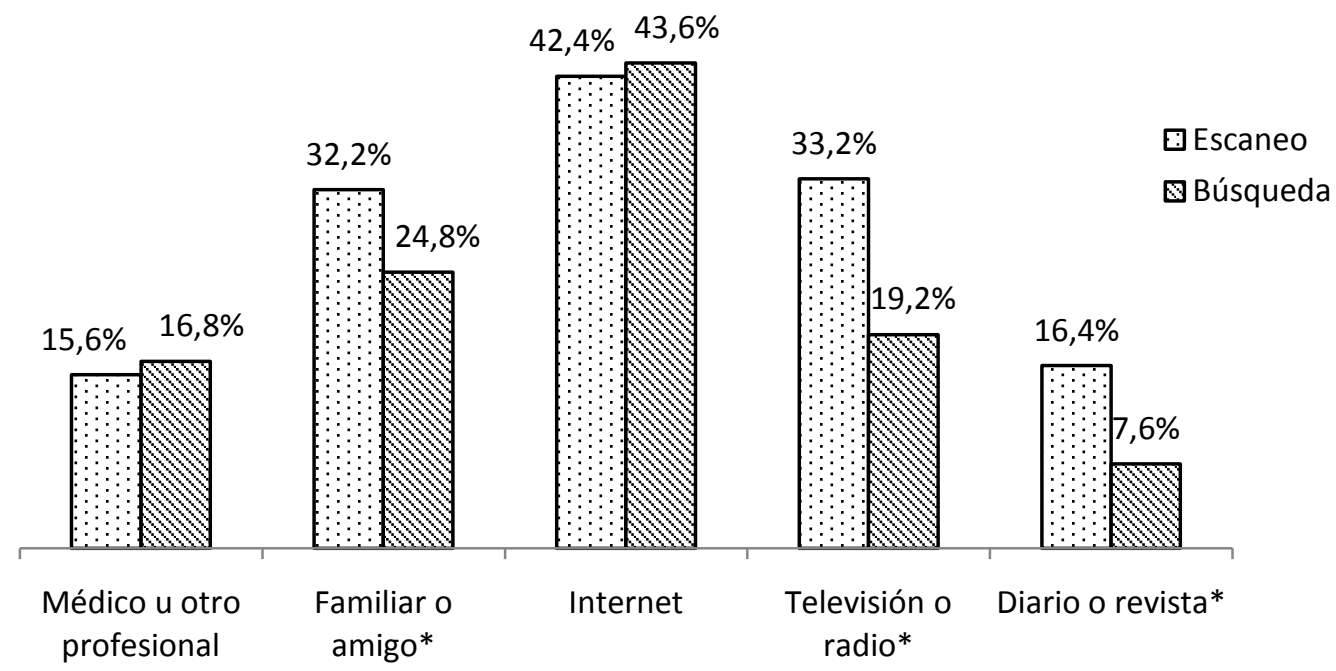

Figura 1. Frecuencia de búsqueda y escaneo de información sobre la práctica de actividad física. ${ }^{*}$ indica diferencia estadísticamente significativa. Fuente: elaboración propia.

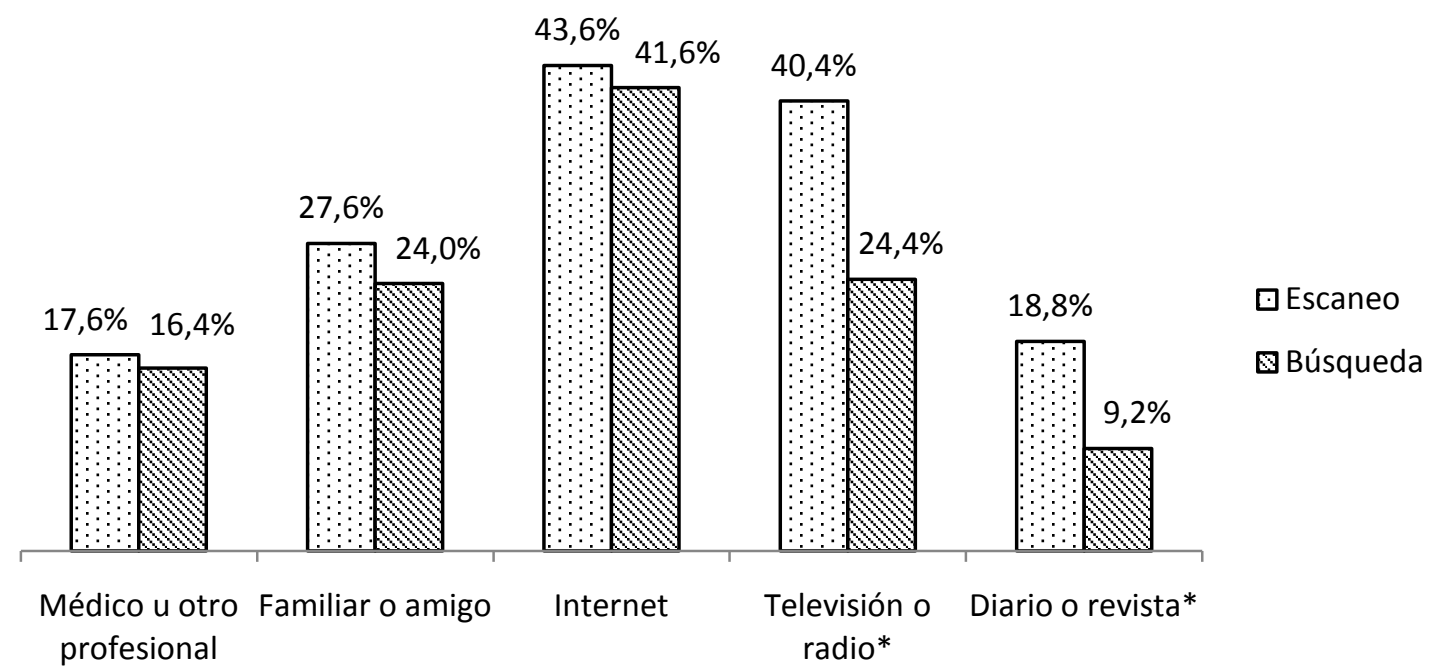

Figura 2. Frecuencia de búsqueda y escaneo de información sobre consumo de frutas y verduras. ${ }^{*}$ indica diferencia estadísticamente significativa. Fuente: elaboración propia.

En referencia a la hipótesis 1, comparadas con los hombres, las mujeres de la muestra presentaron mayores niveles de búsqueda de información sobre actividad física a través de médicos u otros profesionales de la salud $\left(b=-, 79\right.$, Wald $X^{2}(1)=$ 
$4,76, p<, 05)$ y a través de diarios y revistas $\left(b=-2,00\right.$, Wald $\left.X^{2}(1)=8,97, p<, 01\right)$. Con respecto a la información sobre consumo de frutas y vegetales, las mujeres reportan más búsqueda en internet que los hombres $\left(b=-, 61\right.$, Wald $x^{2}(1)=4,66, p<$ ,05). En la búsqueda de información sobre actividad física a través de amigos o familiares, internet o televisión o radio no se hallaron diferencias entre hombres y mujeres. En la búsqueda sobre consumo de frutas y vegetales no se encontraron diferencias entre hombres y mujeres en la obtención de información de parte de médicos u otros profesionales, familiares y amigos, televisión y diarios o revistas.

Adicionalmente, las mujeres reportaron mayor exposición incidental o escaneo de información sobre actividad física $\left(b=-1,79\right.$, Wald $\left.x^{2}(1)=16,46, p<, 05\right)$ y consumo de frutas y vegetales $\left(b=-, 87\right.$, Wald $\left.x^{2}(1)=5,90, p<, 05\right)$ a través de médicos u otros profesionales de la salud comparadas con los hombres. En el caso de la información sobre consumo de frutas y vegetales, las mujeres también reportan más escaneo de la televisión o radio $\left(b=-, 60\right.$, Wald $\left.X^{2}(1)=5,12, p<, 05\right)$. En ninguna de las otras fuentes examinadas se advierten diferencias significativas entre hombres y mujeres.

Con respecto a la hipótesis 2, que refiere a diferencias dadas por el NSE, al examinar la búsqueda de información a través de internet tanto para actividad física (NSE C3: $b=, 97$, Wald $x^{2}(1)=7,70, p<, 01$; NSE C2: $b=, 83$, Wald $x^{2}(1)=5,91, p<$ ,05) como para consumo de frutas y verduras (NSE C3: $b=1,23$, Wald $\chi^{2}(1)=11,87$, $p<, 01$; NSE C2: $b=, 75$, Wald $\left.X^{2}(1)=4,57, p<, 05\right)$ se observa que los grupos medio-bajo y medio ( $\mathrm{C} 3$ y $\mathrm{C} 2$, respectivamente), tienen mayor tendencia a realizar estas acciones comparados con el NSE bajo (D). Lo mismo ocurre para la búsqueda a través de diarios y revistas de información sobre actividad física (NSE C3: $b=2,46$, Wald $X^{2}(1)=5,20, p<, 05$; NSE C2: $b=2,33$, Wald $X^{2}(1)=4,66, p<, 05$ ) y sobre el consumo de frutas y verduras (NSE C3: $b=1,66$, Wald $x^{2}(1)=4,22, p<, 05$; NSE C2: $b=1,84$, Wald $\left.x^{2}(1)=5,40, p<, 05\right)$. En síntesis, solo en el caso de estas dos fuentes de información la hipótesis tiene sustento, pues los entrevistados del grupo socioeconómico más bajo reportaron en menor medida búsqueda de información.

Comparados con el grupo D y el C2, los entrevistados del segmento C3 reportan mayor búsqueda de información sobre ambos temas mediante familiares y amigos (AF: $b=1,68$, Wald $x^{2}(1)=15,16, p<, 001$; FV: $b=1,39$, Wald $x^{2}(1)=12,07$, $p<, 01)$ y mediante televisión y radio en el caso de actividad física (AF: $b=1,65$, Wald $\left.X^{2}(1)=14,89, p<, 001\right)$. Este hallazgo provee soporte para la hipótesis 2 al comparar el segmento C3 con el D, pero no al compararlo con el NSE más alto, el C2.

Se observa que las personas de segmento medio-bajo, comparadas con las del segmento bajo y el medio, reportaron más escaneo de información sobre ambos temas examinados en sus círculos familiares y de amigos (AF: $b=1,13$, Wald $X^{2}(1)=$ $9,12, p<, 01 ; \mathrm{FV}: b=1,00$, Wald $\left.x^{2}(1)=7,20, p<, 01\right)$.

Con respecto a la pregunta 2 , se encontró adicionalmente que entre las mujeres de segmentos medios y medios-bajos la búsqueda de información sobre actividad física a través de diarios y revistas $\left(x^{2}(5)=17,27, p<, 01\right)$ y sobre consumo de frutas y vegetales a través de familiares y amigos $\left(X^{2}(5)=13,03, p<, 05\right)$ es más prevalente que entre los hombres de todos los segmentos y las mujeres del segmento bajo. Entre las mujeres del segmento medio-bajo, la búsqueda sobre el consumo de frutas y verduras a través de internet $\left(x^{2}(5)=18,51, p<, 01\right)$ y televisión o radio $\left(x^{2}(5)\right.$ $=12,29, p<, 05)$, y el escaneo sobre el mismo tema a través de la televisión o radio $\left(x^{2}(5)=15,22, p<, 01\right)$ es más prevalente que en todos los otros grupos. Finalmente, la búsqueda de información sobre consumo de frutas y verduras a través de diarios y revistas $\left(x^{2}(5)=17,27, p<, 01\right)$ y el escaneo sobre el mismo tema entre familiares y amigos $\left(X^{2}(5)=12,38, p<, 05\right)$ es más prevalente entre mujeres del segmento C2.

Sobre la pregunta 3 , que aborda la prevalencia de escaneo por sobre búsqueda en cada NSE, se encontró que en el segmento medio-bajo no había 
diferencias estadísticamente significativas entre búsqueda y escaneo, por lo cual se puede señalar que en ese grupo ambos mecanismos de obtención de información son igualmente prevalentes. En el segmento medio, el escaneo a través de la televisión y radio resultó ser más prevalente que la búsqueda para ambos temas de salud en dichas fuentes (AF: $p<, 01 ; \mathrm{FV}: p<, 05$ ). En el segmento D, el escaneo de información sobre actividad física en televisión y radio $(p<, 001)$ fue más prevalente que la búsqueda. Para todas las otras fuentes examinadas, no se encontraron diferencias significativas entre búsqueda y escaneo en los segmentos C2 y D.

Con respecto a la prevalencia de escaneo por sobre búsqueda entre hombres y mujeres, se encontró que en ambos casos, el escaneo a través de la televisión y radio resultó ser significativamente más prevalente que la búsqueda tanto para información sobre actividad física (mujeres $p<, 001$; hombres $p<, 01$ ) como para información sobre consumo de frutas y verduras (mujeres $p<, 01$; hombres $p<, 05$ ). En el caso de las mujeres, el escaneo sobre consumo de frutas y vegetales a través de diarios y revistas no resultó más prevalente que la búsqueda, pero sí el escaneo a través de dichos medios sobre actividad física $(p<, 05)$, al igual que entre los hombres $(p<, 01)$.

\section{Discusión}

Este estudio presenta un examen detallado a las prácticas de búsqueda y escaneo o exposición incidental a información sobre la práctica regular de actividad física y el consumo de frutas y vegetales en una muestra de adultos de la ciudad de Santiago de Chile. A diferencia de estudios anteriores sobre prácticas de búsqueda y escaneo, que se concentran principalmente en pacientes con cáncer (por ejemplo: Blanch-Hartigan \& Viswanath, 2015), este trabajo se centra en sujetos no diagnosticados con ninguna enfermedad en particular, y en la exposición a información sobre hábitos de vida saludable y de auto-cuidado, tales como la práctica regular de actividad física y el consumo de frutas y verduras.

Consistente con estudios previos (Kelly et al., 2010), se encontró que la búsqueda de información no es más común que el escaneo. El escaneo resulta ser más recurrente que la búsqueda de información a través de algunas de las fuentes examinadas, tales como la televisión o radio. En este caso, la exposición incidental es más frecuente que la búsqueda tanto en hombres como en mujeres. Los diarios o revistas y familiares o amigos fueron también más mencionados como fuentes de escaneo que de búsqueda entre quienes respondieron la encuesta. El resultado respecto de la prevalencia de escaneo por sobre búsqueda en fuentes interpersonales como familiares o amigos respalda lo planteado por autores como Niederdeppe y colegas (2007), que han señalado que, a pesar de que las fuentes interpersonales podrían ser más comúnmente objeto de búsqueda que de escaneo comparadas con las fuentes mediadas, ambos tipos de fuentes pueden ser utilizadas como fuentes de búsqueda o escaneo.

En esa misma línea, resulta interesante que no se haya encontrado diferencias entre búsqueda y escaneo a través de internet, lo que indica que las personas usan este medio para buscar activamente, pero en igual medida se encuentran incidentalmente en la red con información sobre las conductas examinadas en este estudio. Esto último podría deberse a una amplia disponibilidad de información sobre prácticas saludables a través de sitios de redes sociales y portales de internet que favorecería el escaneo sobre estos temas en específico a través de la red, más allá de las motivaciones individuales para buscar activamente.

Tal como estudios en Estados Unidos han dado cuenta (Kelly et al., 2010), en esta muestra de adultos chilenos las mujeres reportan mayores niveles que los hombres tanto de búsqueda como de escaneo de información sobre salud. Sin embargo, dado que este estudio realizó un examen detallado de estas conductas a 
través de diferentes fuentes, es posible advertir que la búsqueda entre mujeres es más prevalente que entre hombres específicamente cuando se considera a médicos u otros profesionales de la salud, diarios o revistas e internet. Esta mayor prevalencia, además, depende del tema sobre el cual se realice la búsqueda, pues no se halló una fuente que fuera más comúnmente usada por las mujeres comparadas con los hombres a través de los dos temas de salud examinados en este estudio. Al igual que para la búsqueda, la mayor prevalencia de escaneo entre las mujeres tiene sustento solo en el caso de algunas fuentes.

La mayor exposición incidental a información sobre actividad física y consumo de frutas y verduras de parte de médicos $u$ otros profesionales de la salud entre las mujeres puede atribuirse a las siguientes causas. En primer lugar, se ha documentado que las mujeres tienden a acudir más al médico (Briscoe, 1987), lo que favorece su exposición por esa vía. Segundo, podría deberse a que los médicos u otros profesionales tiendan a entregar más frecuentemente información sobre hábitos de vida saludable a las mujeres que a los hombres. Finalmente, es posible que las mujeres tengan mayor recordación de la información recibida sobre estos temas de parte de médicos u otros profesionales. Una mayor recordación de la información entregada por un médico u otro profesional de la salud podría estar relacionada con un mayor interés de las mujeres por estos temas, lo que se sustenta en la mayor prevalencia de búsqueda sobre actividad física de parte de estas fuentes entre las mujeres. Estudios futuros podrían examinar estas hipótesis directamente.

Además de las diferencias por género, este estudio da cuenta de que las brechas de búsqueda y escaneo por nivel socioeconómico que estudios en otras poblaciones han encontrado (por ejemplo: Lee, Ramírez, Lewis, Gray, \& Hornik, 2012) se sostienen también en esta muestra. En la presente investigación se hallaron menores niveles de búsqueda de información a través de internet y diarios y revistas en las personas del NSE bajo, lo que puede deberse a un menor nivel de exposición a estos medios debido a barreras para acceder a ellos. En el caso de internet, para poder contar con el servicio es necesario contratarlo y realizar pagos periódicos. Décadas de estudio en el área de la brecha digital han demostrado que, si bien ésta se ha ido cerrando, persisten diferencias de acceso a las nuevas tecnologías de la información entre los segmentos más y los menos ricos de la sociedad (Molina, 2005). De manera similar, el acceso a diarios y revistas está mediado por un pago, a diferencia del acceso a otros medios como la televisión o la radio, para los cuales existen servicios abiertos sin barrera de pago.

El hecho de que existan mayores diferencias según segmento socioeconómico en la búsqueda de información que en el escaneo da cuenta de posibles disparidades en la motivación por acceder a información sobre salud atribuibles al nivel socioeconómico de una persona. En otras palabras, que las personas de segmentos más desfavorecidos se sienten menos motivadas o interesadas en obtener información sobre actividad física y alimentación saludable que las personas de segmentos medios. Estudios en el contexto de la segunda brecha digital dan cuenta de diferencias socioeconómicas al momento de utilizar las tecnologías de la información que muestran distintas preferencias según el nivel educacional de una persona (Bonfadelli, 2002). Otro factor que puede jugar un rol es la desconfianza hacia la información de salud obtenida, que puede desincentivar la búsqueda entre personas de segmentos más bajos (Miller \& Bell, 2012).

Si bien las mujeres escanean y buscan más información sobre salud que los hombres, incluso dentro del subgrupo de las mujeres la tendencia a buscar información está marcada por la diferencia socioeconómica. Las mujeres de segmento bajo escanean y buscan información a través de diversas fuentes menos comúnmente que los hombres y el resto de las mujeres. Con esto, se puede concluir que la condición socioeconómica pesa más que el género para explicar la propensión a 
buscar y escanear información sobre hábitos de vida saludable, lo que subraya la importancia de considerar el factor socioeconómico al estudiar la exposición a información sobre salud.

Este estudio es una primera aproximación a la búsqueda y escaneo de información sobre hábitos de vida saludable, específicamente el consumo de frutas y verduras y la práctica regular de actividad física, en una muestra de adultos en el área metropolitana de Chile. La presente investigación es un aporte al campo de la comunicación de la salud, pues es una de las primeras en examinar estas conductas en una muestra de pacientes sanos fuera de los Estados Unidos. Además, los resultados de este estudio dan cuenta de brechas relativas al género y al nivel socioeconómico en cuanto a la prevalencia de la búsqueda y escaneo de información, que podrían tener un efecto directo tanto en el conocimiento que los individuos adquieren sobre los temas de salud (Zhao, 2014), en los antecedentes del comportamiento, como actitudes, percepciones normativas e intenciones (Lee et al., 2016), y en la práctica de conductas de auto-cuidado en salud (Ramírez et al., 2013).

A pesar de sus contribuciones, este estudio está limitado debido a que la muestra no es representativa a nivel nacional o regional. No obstante, no es el propósito de este estudio hacer generalizaciones de estos resultados, sino más bien presentar comparaciones entre hombres y mujeres y entre niveles socioeconómicos, con la limitación de que estas conclusiones solo son aplicables a la muestra estudiada. A pesar de la falta de representatividad, el hecho de que se trate de una encuesta cara a cara y en lugares de acceso público le añade diversidad a la muestra y valor a los datos obtenidos, por sobre una encuesta telefónica o a través de internet. Futuros estudios deberán trabajar con muestras representativas a nivel nacional o regional.

Este proyecto ha sido realizado con apoyo del departamento de Comunicación de la Universidad de Illinois en Urbana-Champaign, Estados Unidos. 


\section{Referencias}

Askelson, N. M., Campo, S., \& Carter, K. D. (2011). Completely isolated? Health information seeking among social isolates. Health Education \& Behavior, 38(2), 116-122. doi: 10.1177/1090198110369055

Attewell, P. (2001). The first and second digital divides. Sociology of Education, 74(3), 252. doi: $10.2307 / 2673277$

Beaudoin, C. E., \& Hong, T. (2011). Health information seeking, diet and physical activity: An empirical assessment by medium and critical demographics. International. Journal of Medical Informatics, 80(8), 586-595. doi: http://dx.doi.org/10.1016/j.ijmedinf.2011.04.003

Blanch-Hartigan, D., \& Viswanath, K. (2015). Socioeconomic and sociodemographic predictors of cancer-related information sources used by cancer survivors. Journal of Health Communication, 20(2), 204-210. doi: 10.1080/10810730.2014.921742

Bonfadelli, H. (2002). The internet and knowledge Gaps: A theoretical and empirical investigation. European Journal of Communication, 17(1), 65-84. doi: $10.1177 / 0267323102017001607$

Briscoe, M. E. (1987). Why do people go to the doctor? Sex differences in the correlates of GP consultation. Social Science \& Medicine, 25(5), 507-513. doi: http://dx.doi.org/10.1016/0277-9536(87)90174-2

Galarce, E. M., Ramanadhan, S., Weeks, J., Schneider, E. C., Gray, S. W., \& Viswanath, K. (2011). Class, race, ethnicity and information needs in posttreatment cancer patients. Patient Education and Counseling, 85(3), 432-439. doi: http://dx.doi.org/10.1016/j.pec.2011.01.030

Guillou, M., Carabantes, J., \& Bustos, V. (2011). Disponibilidad de médicos y especialistas en Chile [Availability of physicians and specialists in Chile]. Revista Médica de Chile, 139, 559-570.

Hornik, R., Parvanta, S., Mello, S., Freres, D., Kelly, B., \& Schwartz, J. S. (2013). Effects of scanning (routine health information exposure) on cancer screening and prevention behaviors in the general population. Journal of Health Communication, 18(12), 1422-1435. doi: 10.1080/10810730.2013.798381

Kelly, B., Hornik, R., Romantan, A., Schwartz, J. S., Armstrong, K., DeMichele, A., . . . Wong, N. (2010). Cancer information scanning and seeking in the general population. Journal of Health Communication, 15(7), 734-753. doi: 10.1080/10810730.2010.514029

Kelly, B. J., Niederdeppe, J., \& Hornik, R. C. (2009). Validating measures of scanned information exposure in the context of cancer prevention and screening behaviors. Journal of Health Communication, 14(8), 721-740. doi: 10.1080/10810730903295559

Lee, C.-j. (2009). The interplay between media use and interpersonal communication in the context of healthy lifestyle behaviors: Reinforcing or substituting? Mass Communication and Society, 13(1), 48-66. doi: 10.1080/15205430802694869

Lee, C.-j., Ramírez, A. S., Lewis, N., Gray, S. W., \& Hornik, R. C. (2012). Looking beyond the internet: Examining socioeconomic inequalities in cancer information seeking among cancer patients. Health Communication, 27(8), 806817. doi: 10.1080/10410236.2011.647621

Lee, C.-J., Zhao, X., \& Pena-y-Lillo, M. (2016). Theorizing the pathways from seeking and scanning to mammography screening. Health Communication, 31(1), 117128. doi: 10.1080/10410236.2014.942769

Miller, L. M. S., \& Bell, R. A. (2012). Online health information seeking: The influence of age, information trustworthiness, and search challenges. Journal of Aging and Health, 24(3), 525-541. doi: 10.1177/0898264311428167 
Ministerio de Salud (2010). Encuesta Nacional de Salud ENS Chile 2009-2010 [Chile 2009-2010 National Health Survey]. Retrieved from: http://web.minsal.cl/portal/url/item/bcb03d7bc28b64dfe040010165012d23.pdf

Molina, F. J. F. (2005). Brecha e inclusión digital en Chile: Los desafíos de una nueva alfabetización. [ Gap and digital inclusion in Chile: The challenges of a new literacy] Comunicar: Revista Científica Iberoamericana de Comunicación y Educación(24), 77-84.

Niederdeppe, J., Hornik, R. C., Kelly, B. J., Frosch, D. L., Romantan, A., Stevens, R. S., . . . Schwartz, J. S. (2007). Examining the dimensions of cancer-related information seeking and scanning behavior. Health Communication, 22(2), 153167. doi: $10.1080 / 10410230701454189$

Ramanadhan, S., \& Viswanath, K. (2006). Health and the information nonseeker: A profile. Health Communication, 20(2), 131-139. doi: 10.1207/s15327027hc2002_4

Ramírez, A. S., Freres, D., Martinez, L. S., Lewis, N., Bourgoin, A., Kelly, B. J., . . . Hornik, R. C. (2013). Information seeking from media and family/friends increases the likelihood of engaging in healthy lifestyle behaviors. Journal of Health Communication, 18(5), 527-542. doi: 10.1080/10810730.2012.743632

Rutten, L. J. F., Squiers, L., \& Hesse, B. (2006). Cancer-related information seeking: Hints from the 2003 Health Information National Trends Survey (HINTS). Journal of Health Communication, 11(sup001), 147-156. doi: 10.1080/10810730600637574

Shim, M. (2008). Connecting internet use with gaps in cancer knowledge. Health Communication, 23(5), 448-461. doi: 10.1080/10410230802342143

Shim, M., Kelly, B., \& Hornik, R. (2006). Cancer information scanning and seeking behavior is associated with knowledge, lifestyle choices, and screening. Journal of Health Communication, 11(sup001), 157-172. doi: 10.1080/10810730600637475

Viswanath, K., \& Ackerson, L. K. (2011). Race, ethnicity, language, social class, and health communication inequalities: A nationally-representative cross-sectional study. PLOS ONE, 6(1), 1-8. doi: 10.1371/journal.pone.0014550

Viswanath, K., \& Emmons, K. M. (2009). Health communication and communication inequalities in addressing cancer disparities. In H. K. Koh (Ed.), Toward the Elimination of Cancer Disparities (pp. 277-298). New York, NY: Springer.

Zhao, X. (2014). Relationships between sources of health information and diabetes knowledge in the U.S. Hispanic population. Health Communication, 29(6), 574585. doi: 10.1080/10410236.2013.784937 